\title{
Genesis
}

Manuscrits - Recherche - Invention

\section{La valorisation des archives du Roald Dahl Museum and Story Centre : de la conservation à l'exploitation didactique}

Entretien avec Rachel White

\section{Christine Collière-Whiteside}

\section{OpenEdition}

Journals

\section{Édition électronique}

URL : http://journals.openedition.org/genesis/4119

DOI : 10.4000/genesis.4119

ISSN : 2268-1590

\section{Éditeur :}

Presses universitaires de Paris Sorbonne (PUPS), Société internationale de génétique artistique littéraire et scientifique (SIGALES)

\section{Édition imprimée}

Date de publication : 1 juin 2019

Pagination : 109-115

ISSN : 1167-5101

\section{Référence électronique}

Christine Collière-Whiteside, « La valorisation des archives du Roald Dahl Museum and Story Centre : de la conservation à l'exploitation didactique », Genesis [En ligne], 48 | 2019, mis en ligne le 01 juin 2020, consulté le 24 janvier 2021. URL : http://journals.openedition.org/genesis/4119; DOl : https:// doi.org/10.4000/genesis.4119 


\title{
La valorisation des archives du Roald Dahl Museum and Story Centre : de la conservation à l'exploitation didactique Entretien avec Rachel White*
}

\author{
Propos recueillis par Christine Collière-Whiteside
}

\begin{abstract}
Rachel White est responsable des collections et archiviste au Roald Dahl Museum and Story Centre à Great Missenden au nord-ouest de Londres depuis 2012. Inauguré en 2005, ce musée s'adresse aux enfants de 6 à 12 ans, mais aussi aux adultes, et comporte trois galeries interactives. Il abrite les archives Roald Dahl, une des plus complètes parmi les archives littéraires consacrées à un seul auteur.
\end{abstract}

Christine Collière-Whiteside - Pourriez-vous nous donner une idée de l'étendue et de la nature des documents qui composent les archives?

Rachel White - Le fonds inclut les archives littéraires de Roald Dahl, qui comprennent ses documents de travail (ses carnets d'idées [«Ideas Books »], ses manuscrits et tapuscrits, les correspondances avec ses éditeurs) et les œuvres publiées (livres et magazines), ainsi que ses papiers personnels : sa correspondance, les objets de sa cabane d'écrivain et des photographies ayant trait à sa vie et à son œuvre. Nous conservons aussi des vêtements et des objets qui lui appartenaient, ainsi que des documents secondaires tels que des thèses universitaires, des accessoires, costumes et publications éphémères relatives aux adaptations de ses œuvres à l'écran, des traductions de ses œuvres ainsi que les documents concernant la création du musée lui-même.

Il est important de noter que ces papiers sont ceux que Roald avait choisi de garder : nous savons qu'il a brûlé une partie de ses documents de travail, et certains des premiers brouillons de ses œuvres sont trop «propres» (sans ratures ou ajouts) pour être réellement les premières ébauches. Je pense qu'il s'agit plutôt de versions au propre des premiers brouillons, que Dahl avait gardées pour s'y référer plus tard.

Les manuscrits ont été conservés dans l'ordre où Roald les avait rangés, mais ils ont été placés dans des dossiers sans acide. Ils sont presque tous rédigés sur du papier jaune ligné au format «legal», en général au crayon lorsqu'ils sont rédigés à la main, parfois annotés au stylo-bille. Ils sont accompagnés des tapuscrits des brouillons, qui sont parfois eux-mêmes devenus des brouillons lorsque Roald les a retravaillés, là aussi au crayon ou au stylo.

Une grande partie des brouillons montrent comment il travaillait : on y trouve des exemples de mots biffés ou remplacés, de notes marginales, de phrases ou de paragraphes entiers insérés à l'aide de «bulles » ou de flèches indiquant le point d'insertion dans le texte. Il lui arrivait aussi de réunir des parties de texte à l'aide de ruban adhésif. Nous avons aussi deux documents qui sont différents : les maquettes illustrées des deux premières versions de Fantastic Mr Fox.

C. C.-W. - Comment ce fonds a-t-il été réuni? L'idée de rendre ses archives disponibles de cette manière avait-elle été évoquée par Roald Dahl?

$R$. W. - La plupart des documents ont été rassemblés par Roald Dahl de son vivant et l'ensemble des documents a été légué au musée par sa veuve Liccy Dahl à la mort de l'auteur. D'après ce que m'ont dit mes collègues, Roald souhaitait que ses archives restent au Royaume-Uni, et Liccy a fait en sorte de respecter cette volonté. J'ignore dans quelle mesure Roald souhaitait que ce fonds soit rendu accessible aux chercheurs ou au grand public; il faudrait vérifier cela auprès de Liccy mais nous ne sommes pas en contact régulier avec elle.

(*) Merci à Natalie Wallace, responsable de l'équipe pédagogique, pour les informations complémentaires concernant le programme destiné aux écoles. 
C. C.-W. - Les archives concernant ses nouvelles pour adultes sont bien moins riches que les dossiers concernant ses livres pour enfants. Que contiennent les archives à propos de la genèse des nouvelles?

$R$. W. - Nous possédons la correspondance avec les éditeurs concernant les nouvelles. Elle n'a pas été cataloguée document par document, mais nous avons décrit chacun des dossiers.

C. C.-W. - Lorsque Dahl a commencé à écrire pour les enfants, il écrivait d'abord le texte et ne commençait à chercher un illustrateur qu'une fois le texte achevé. Y a-t-il des cas où Dahl a modifié son texte suite à un échange avec un artiste autre que Quentin Blake?

$R$. W. - Non, je ne crois pas. Il a correspondu avec Nancy Ekholm Burkert à propos des illustrations de James mais il s'agissait plutôt de la façon dont elle interprétait son texte, Roald n'a pas fait de modifications suite à cet échange.

C. C.-W. - Ce n'est que lorsqu'il a travaillé sur The Enormous Crocodile avec Quentin Blake (c'est-à-dire lors de leur quatrième collaboration) qu'un véritable travail de création à deux a commencé, avant que le texte soit terminé. Quentin Blake a raconté comment il venait à Gypsy House et dessinait pendant que Dahl lui parlait de l'histoire qu'il était en train d'écrire et comment des idées ou des modifications surgissaient parfois de leurs discussions, notamment le costume de Miss Trunchbull ou du BFG : on connaît la célèbre histoire du colis envoyé par la poste à Quentin Blake, qui contenait la propre sandale norvégienne de Dahl en guise de suggestion pour les chaussures du BFG. $Y$ a-t-il des traces de cette collaboration dans les documents présents dans l'archive?

R. W. - Pas directement, mais Roald a raconté l'histoire de la genèse de The Giraffe and the Pelly and $M e^{1}$. Il voulait écrire une autre histoire pour de très jeunes enfants, mais n'avait ni personnages ni scénario. Il demanda donc à Quentin de lui donner le nom de trois animaux qu'il aimerait dessiner, et Quentin lui répondit qu'il aimerait essayer de dessiner une girafe, qu'il avait eu plaisir à dessiner le singe dans The Enormous Crocodile et qu'il serait amusant de dessiner un pélican, à cause de son bec. Quentin demanda à Roald s'il avait déjà une histoire et la réponse fut : «Non, pas encore.»

C. C.-W. - Comment le musée a-t-il été conçu ? Y a-t-il eu beaucoup de changements au cours de ses treize ans d'existence? Et pourriez-vous nous parler des différentes façons dont vous avez valorisé les documents à votre disposition?

$R$. W. - Le musée a été créé pour accueillir les archives de Roald Dahl, mais aussi pour mieux faire connaître sa vie et offrir aux gens un lieu où explorer leur propre créativité. C'est un objectif sur lequel nous nous sommes focalisés de plus en plus au cours des dix dernières années depuis l'ouverture du musée en 2005, comme on peut le voir dans notre déclaration d'intention : "Que tous ceux qui visitent le Roald Dahl Museum and Story Centre comprennent comment l'œuvre de Roald Dahl peut constituer une clef pour révéler les histoires que nous avons tous en nous. » Nous venons juste de lancer une étude sur la façon dont les visiteurs s'emparent du musée afin de réfléchir aux moyens de mieux accueillir les familles, c'est-à-dire des groupes hétérogènes du point de vue de l'âge, pour qu'elles profitent du musée ensemble, plutôt que d'être un musée conçu uniquement pour les enfants, même si les enfants constituent notre public principal.

Mettre en valeur les documents des archives est parfois délicat : le contenu des manuscrits de Roald Dahl est fascinant et passionnant mais ce ne sont pas des documents d'un abord facile, car ils sont écrits à la main sur du papier jaune, ce qui peut être difficile à déchiffrer, et il n'est donc pas aisé de susciter l'intérêt de nos visiteurs, en particulier des enfants. Nous changeons les documents exposés en vitrine tous les trois mois, et nous choisissons des thèmes et des sujets qui correspondent à l'actualité culturelle. Par exemple, notre thème général au cours des mois d'avril-mai cette année était Roald Dahl et l'aviation, en lien avec les célébrations des 100 ans de la RAF. Nous avons proposé aux visiteurs des ateliers et des jeux de piste sur le thème du vol, à la fois dans les romans de Dahl (James and the Giant Peach, The Minpins, The Roly-Poly Bird, The Gremlins et The Great Glass Elevator) et dans la vie de Roald lorsqu'il était pilote

1. Dans une lettre pour le bulletin d'information de Tom Maschler datée de 1985, reprise dans le Roald Dahl Treasury, London, Jonathan Cape, 1997, «Creating characters», p. 62-63. 
de chasse dans la RAF pendant la Seconde Guerre mondiale. Le choix des documents exposés reflétait ces thèmes. Ainsi, nous avons choisi d'exposer des documents des archives concernant son texte autobiographique Going Solo, en particulier les passages concernant sa participation à la bataille d'Athènes en 1941. Nous avons aussi organisé l'exposition d'objets qu'il avait utilisés pendant cette période : sa veste et son casque de pilote ainsi que ses cartes de navigation.

Lorsque nous exposons des documents des archives, les légendes expliquent la nature de chaque document et ce qui en fait l'intérêt ou l'importance. Les éléments exposés sont choisis de façon à ce qu'ils soient en lien les uns avec les autres et qu'ils racontent une histoire, dans la mesure du possible, afin que les visiteurs apprennent quelque chose à propos d'une œuvre, d'une partie du processus créateur de Roald Dahl ou encore d'un événement de sa vie qui a inspiré ses histoires. Les manuscrits difficiles à déchiffrer sont accompagnés d'une transcription afin que les visiteurs puissent en tirer le meilleur profit. Nous nous efforçons également d'inclure des éléments visuels - objets ou photographies - afin que les pièces exposées ne soient pas toutes du même type. Cette année, nous avons aussi testé l'emploi de repères visuels à côté des manuscrits : par exemple, pour les documents concernant les insectes dans James and the Giant Peach nous avons placé de petites images d'insectes à côté des passages concernés. Cela permettait d'attirer le regard vers le manuscrit, d'apporter de la couleur et de susciter la curiosité, notamment des enfants non lecteurs qui n'auraient pas été intéressés par les manuscrits exposés.

C. C.-W. - Voilà qui est passionnant. C'est très vrai. Nous nous sommes rendu compte que les auteurs-illustrateurs qui tiennent des blogs ou qui ont un site web y publient plus souvent des croquis ou des dessins que des exemples de brouillons et de textes en cours d'écriture, que nous avons beaucoup plus de mal à trouver. Le succès des expositions de la galerie d'art The House of Illustration à Londres ou du Centre international de l'illustration à Strasbourg témoigne de la popularité des projets autour de la littérature pour la jeunesse, du moins en ce qui concerne les illustrations, mais intéresser le grand public aux textes s'avère plus difficile. Pourriez-vous nous donner des exemples de la façon dont vous sélectionnez des documents pour construire un fil narratif dans une exposition?
R. W. - Ce serait par exemple pour The Twits ou The BFG, lorsque nous pouvons exposer une des toutes premières idées de Roald pour une histoire - une scène ou un personnage - dans ses carnets d'idées, puis montrer le développement de l'histoire et du roman au fil des premiers brouillons, de la correspondance avec ses éditeurs, des références au livre ou aux personnages dans les conférences ou les entretiens de Roald, pour finir par des éléments post-éditoriaux tels que les traductions ou la réception des œuvres.

Nous pouvons aussi choisir un concept comme l'humour ou la poésie dans ses œuvres et trouver des exemples tirés de sa vie pour l'illustrer. Par exemple, nous avons en projet une exposition autour du caractère puéril de l'humour de Dahl, qui réunira des lettres-poissons d'avril écrites à sa mère, les blagues sur les vents et les « crépitages ${ }^{2}$ » dans The BFG, des documents illustrant le sens inné de Roald pour le grotesque et pour ce qui faisait rire les enfants, et la conviction qu'il avait d'être capable de se mettre dans la peau d'un enfant et de se souvenir de ce qu'ils trouvent amusant.

C. C.-W. - Depuis 2013, vous avez aussi tiré parti du blog sur le site du musée pour valoriser les archives. Vous ne vous contentez pas d'y présenter quelques-uns des documents les plus mémorables. De nombreux billets incluent des photos de pages tirées des brouillons et les commentent de façon détaillée. Je me souviens par exemple de cette liste de mots inventés tirée des avant-textes du BFG (fig. 1).

$R$. W. - Les blogs sont un autre moyen de faire découvrir les archives à un public plus large. Dans la mesure du possible, nous faisons en sorte que les pièces exposées dans les vitrines et les billets publiés sur le blog soient en lien avec les thèmes explorés au même moment dans le musée. Souvent, les entrées du blog nous permettent d'aller plus loin dans l'analyse qu'il n'est possible de le faire dans les légendes présentes dans les vitrines. Ainsi, dans l'entrée du 21 décembre 20173, nous avons montré comment Dahl utilisait les descriptions des yeux de ses personnages pour suggérer leur personnalité, en nous appuyant à la fois sur les œuvres, les brouillons et la technique de Dahl qui consistait à

\footnotetext{
2. «Whizzpopping», dans la traduction de Jean-François Ménard. 3. < http://www.roalddahl.com/blog/2017/december/marvellous-magicand-hidden-powers $>$.
} 


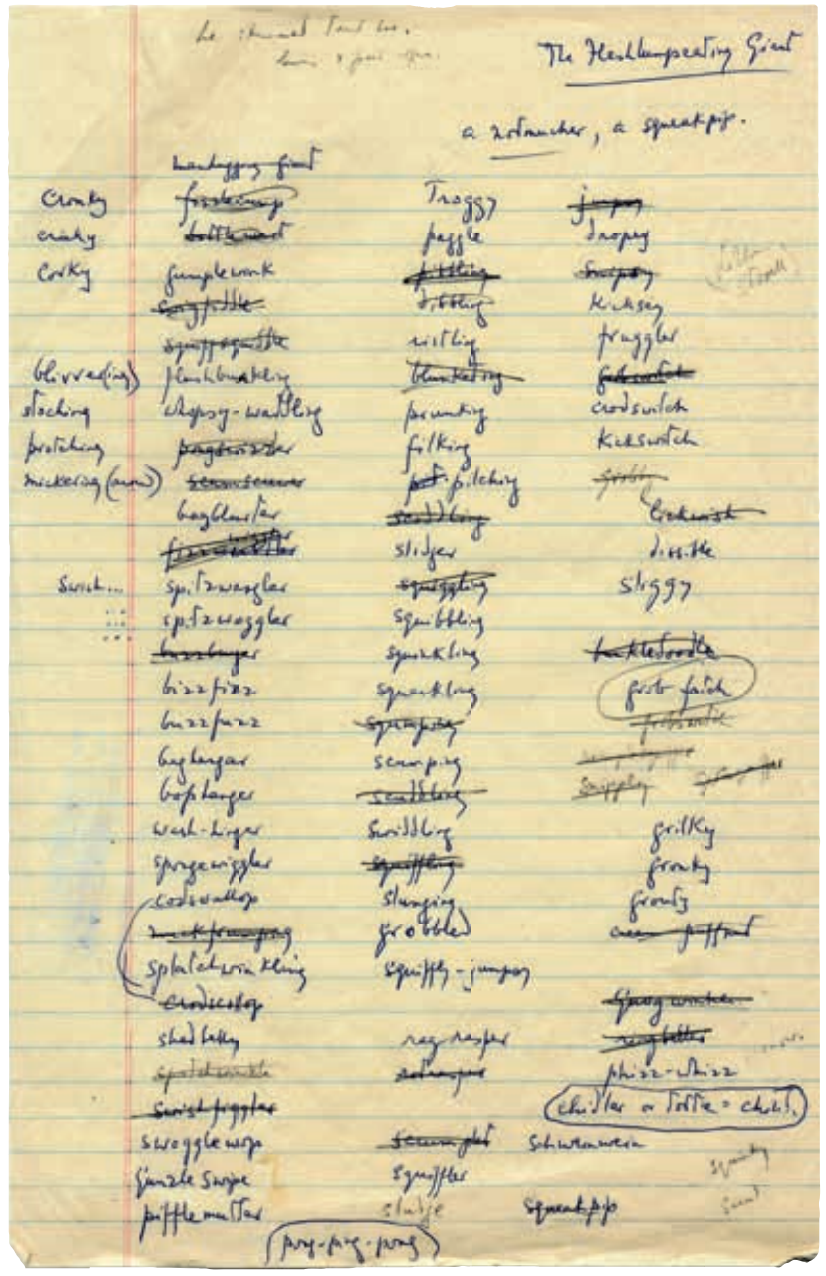

Fig. 1 : Roald Dahl, liste de mots inventés, avant-texte de The BFG, RD/2/19/1, 1981

(C) Roald Dahl Story Company Ltd

découper des yeux dans des photos trouvées dans des magazines et des journaux pour s'en inspirer. De même, il y a une entrée sur «les bestioles et les méchants ${ }^{4}$, qui exploite des exemples tirés de différents manuscrits correspondants à plusieurs de ses œuvres. La liste de 300 mots que Roald a inventés pour le $B F G^{5}$ n'est qu'un des avant-textes de ce roman que le blog aborde : un autre billet présente la page de son carnet d'idées où apparaît pour la première fois le personnage d'un géant qui vole les pensées et les rêves des gens et les collectionne dans des pots de verre 6 . Quelques mois plus tard, un autre billet explique comment le $B F G$ est d'abord apparu dans Danny the Champion of the World, en s'appuyant sur des brouillons de Danny ${ }^{7}$. Plusieurs autres entrées parlent des versions successives de l'histoire qui deviendrait Charlie and the chocolate factory (fig. 2) ou expliquent comment Matilda était à l'origine une méchante petite fille. Parfois nous utilisons des reproductions de

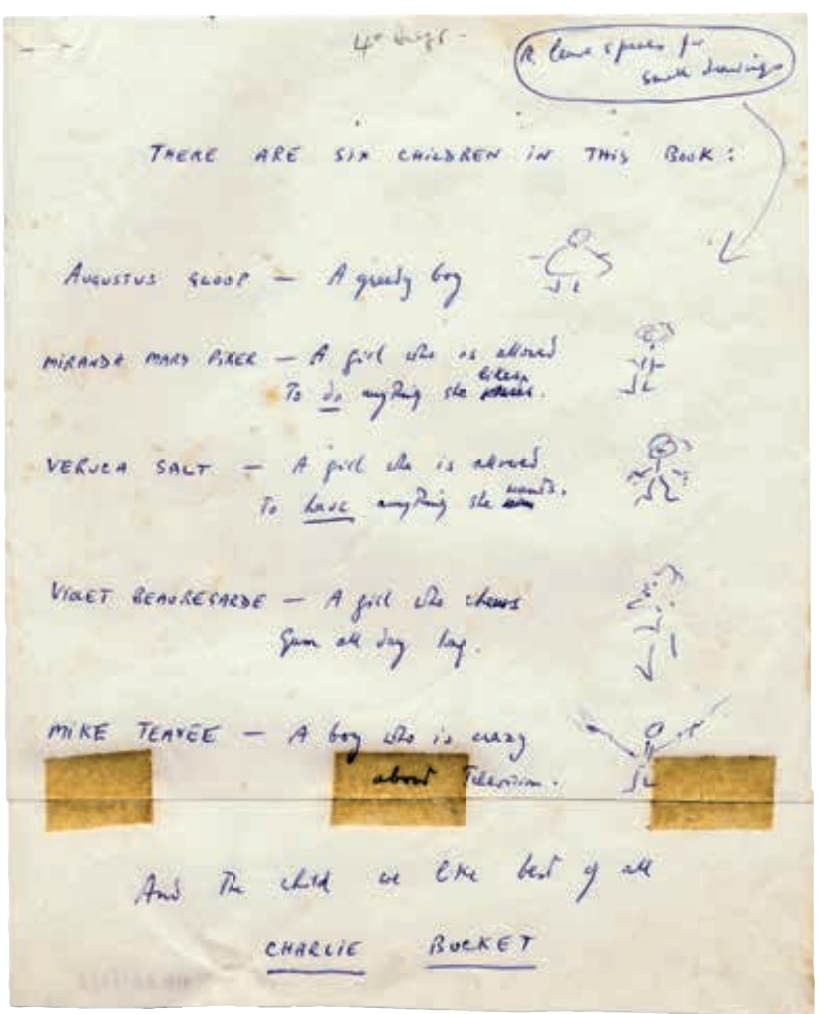

Fig. 2 : Roald Dahl, liste de personnages dans le manuscrit intitulé «Charlie and the chocolate factory » et marqué «4th draft», RD/2/7/5, 1962.

(c) Roald Dahl Story Company Ltd

passages des manuscrits de Dahl pour illustrer le thème d'une entrée du blog, comme celle sur «les super-papas dans les romans de Roald Dahl ${ }^{\circledR}$. Une des entrées les plus populaires, avec 6945 vues à ce jour, est celle qui parle de la cabane d'écrivain de Roald?.

4. <http://www.roalddahl.com/blog/2017/october/beasties-and-baddiesin-roald-dahls-books $>$, publié le 26 octobre 2017.

5. <http://www.roalddahl.com/blog/2016/july/gobblefunk-around-withwords $>$, publié le 27 juillet 2016 .

6. <http://www.roalddahl.com/blog/2016/july/the-first-appearance-ofthe-bfg >, publié le 18 juillet 2016 .

7. <http://www.roalddahl.com/blog/2016/november/the-bfg-and-dannythe-champion-of-the-world >, publié le 17 novembre 2016.

8. <http://www.roalddahl.com/blog/2016/june/top-dads-in-roald-dahlbooks $>$, publié le 17 juin 2016.

9. <http://www.roalddahl.com/blog/2017/may/inside-roald-dahlswriting-hut $>$. 


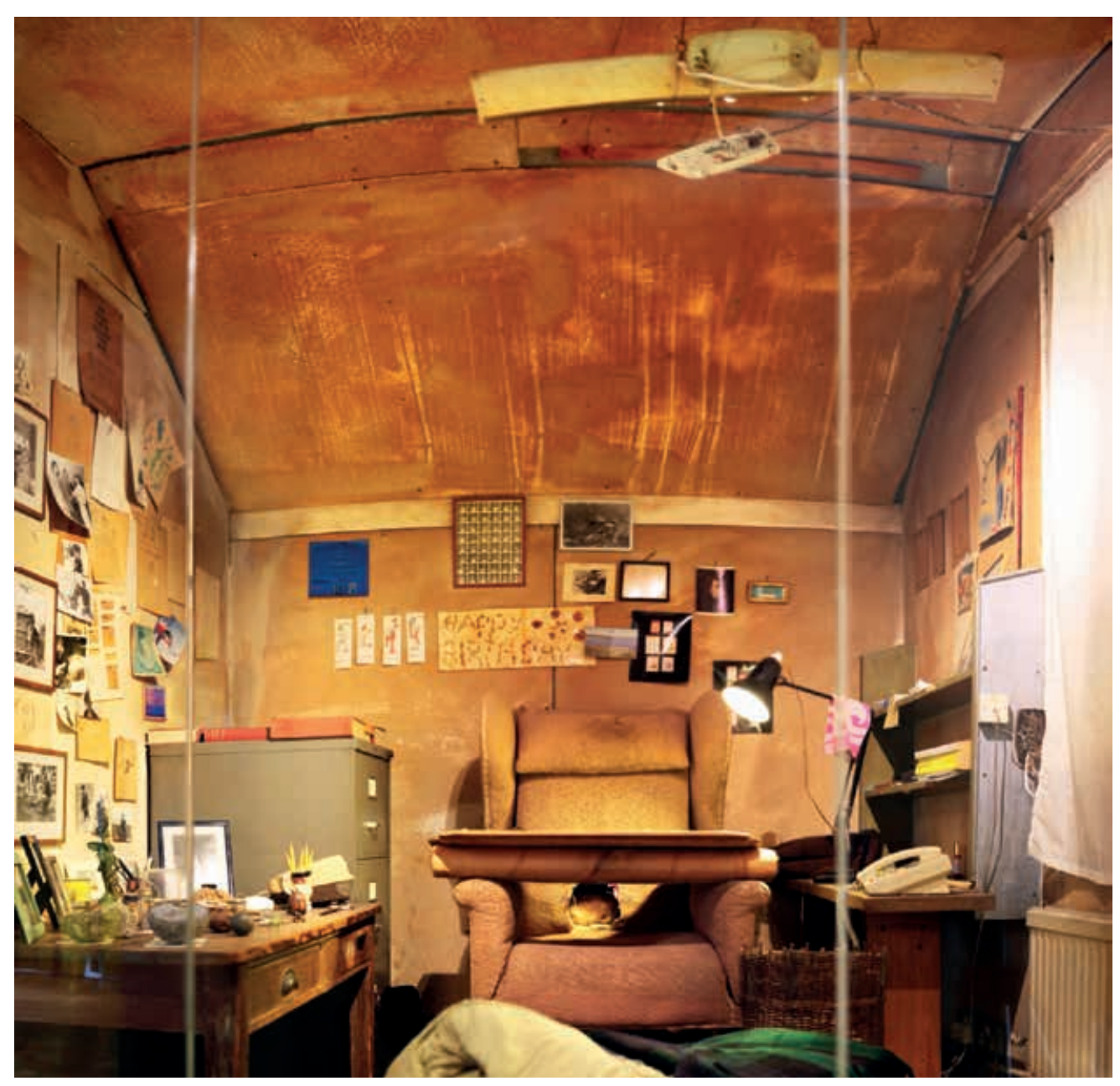

Fig. 3 : Intérieur de la cabane d'écrivain de Roald Dahl. () The Roald Dahl Museum and Story Centre

C. C.-W. - En effet, en 2012, l'intérieur de la «cabane» de Dahl (fig. 3), avec le fauteuil qu'il avait adapté lui-même à ses besoins et tous les objets idiosyncratiques qu'il y gardait à portée de main pour les contempler et s'en inspirer ont été soigneusement conservés et méthodiquement transférés de son jardin vers une nouvelle galerie dans le musée. Un petit livre a même été publié, où tous les objets de sa cabane sont reproduits et soigneusement numérotés ${ }^{10}$. Il s'en dégage à la fois la rigueur d'une description de site archéologique, la ferveur qu'inspirent des reliques sacrées, mais aussi tout simplement un grand plaisir. C'est un projet très différent de ce que l'on voit dans les maisons d'écrivains célèbres que l'on peut visiter et qui nous en disent souvent davantage sur la façon dont les gens vivaient à leur époque que sur la personne de l'auteur. Le fait que ce soit centré autour de la cabane de l'écrivain plutôt que de sa maison (pour des raisons évidentes puisque sa famille y vit encore) en fait un projet unique, qui s'intéresse avant tout à Dahl en tant qu'auteur et non en tant que personne privée, tout en étant à la fois très concret et très intime. Comment ce projet a-t-il vu le jour? Était-ce prévu dès la création du musée? Les enfants peuvent-ils toujours s'asseoir dans le fauteuil?

$R$. W. - À ma connaissance, la cabane d'écrivain a été transférée principalement parce qu'elle risquait de se dégrader là où elle se trouvait à Gypsy House, et que la famille et les administrateurs du musée ne voulaient pas prendre le risque de perdre un artefact si important. L'idée a toujours été de la préserver dans l'état où Roald l'avait conservée durant sa vie, et tout ce qui s'y trouvait a donc été inventorié avec soin avant de déplacer quoi que ce soit.

10. Inside Dahl's Writing Hut, Roald Dahl Museum and Story Centre, 2012. 
Nous n'autorisons pas les enfants à s'asseoir dans le fauteuil de la cabane elle-même, pour des raisons de conservation et de sécurité, mais les enfants (et les adultes) peuvent s'asseoir dans le fauteuil qui se trouve dans la réplique de la cabane située dans la maison des histoires («Story Centre»).

C. C.-W. - Dahl avait l'habitude d'aller dans les écoles pour parler aux enfants, n'est-ce pas? Existe-t-il dans les archives des traces de ces rencontres? Sait-on s'il apportait des manuscrits pour les montrer aux élèves ou venait-il surtout parler et répondre aux questions des enfants? A-t-il jamais collaboré avec des enseignants ou des élèves d'une manière ou d'une autre?

R. W. - Oui, il allait dans les écoles autour de Great Missenden parler aux élèves et nous avons certains brouillons de ses causeries dans les archives. Il parlait de son métier d'écrivain : d'où lui venaient ses idées, le contexte de certains de ses romans, comment les histoires et les personnages s'étaient développés et comment il était devenu écrivain pour commencer. Il apportait parfois des objets de sa cabane pour les montrer aux élèves, comme la Boule d'Emballages de Chocolats, mais à ma connaissance il n'a jamais apporté ses brouillons. Il n'y avait pas de collaboration à proprement parler, mais nous savons par sa correspondance avec ses éditeurs qu'il tenait compte de ce que les enfants lui disaient et qu'il a effectué des modifications en conséquence. Par exemple, il a modifié un passage de The Twits après en avoir lu un chapitre à des enfants qui ont remarqué une erreur logistique.

C. C.-W. - Comment le projet de la «maison des histoires» s'est-il développé? Était-elle présente dès la conception du musée? Pourriez-vous décrire les différents coins de cette partie du musée et les activités qui sont proposées aux groupes scolaires qui viennent avec leurs enseignants?

$R$. W. - L'idée de la maison des histoires était de donner l'occasion aux visiteurs, en particulier aux enfants, de créer leurs propres histoires et leurs propres personnages en utilisant différents médias : des films d'animation, des collages, en se déguisant, avec des mots magnétiques pour créer des poèmes. L'un des principes directeurs de la création du musée était d'encourager les visiteurs à explorer leurs propres vies comme inspiration pour raconter des histoires.
En s'appuyant sur les archives, notre équipe pédagogique a mis en place un programme pour les écoles dont l'objectif est d'explorer comment Dahl travaillait pour améliorer ses textes : comment il révisait et réécrivait ses textes, comment il les lisait de manière critique, et comment il créait ses personnages à partir de sa propre expérience. Après avoir examiné les outils de Dahl et ses techniques d'écriture ou de réécriture dans le musée, les élèves sont invités à développer un personnage dans la maison des histoires à partir d'une phrase écrite par Dahl qu'ils doivent modifier, ou en utilisant les photos des yeux découpées par Dahl dans des magazines, ou, pour les séances plus longues, en jouant à être des relecteurs clandestins et à réécrire des passages des brouillons de Dahl. Nous montrons aux élèves plus âgés (correspondant à des classes du CM1 à la $5 \mathrm{e}$ ) des réécritures de passages plus longs tirés des avant-textes de Charlie ou de James, et nous les amenons à utiliser les techniques ainsi découvertes pour modifier leurs propres textes, ou encore nous leur montrons comment utiliser des éléments de la vie réelle, tels que des lettres, des titres de journaux ou des extraits de documents pour rendre un texte biographique plus mémorable, comme Dahl l'a fait dans Boy ou Going Solo.

C. C.-W. - Quel accueil les enseignants ont-ils réservé à ce programme pédagogique?

$R$. W. - Le programme proposé aux écoles est très demandé, nous affichons presque complet chaque année (environ $97 \%$ ), ce qui est exceptionnel pour un musée de notre taille. Nous l'avons remanié l'année dernière afin de mettre davantage l'accent sur nos collections, et nous avons eu des retours très positifs. Nous venons juste de recevoir le prix Sandford, qui récompense les initiatives pédagogiques valorisant le patrimoine culturel.

C. C.-W. - Existe-t-il d'autres musées ou d'autres archives littéraires qui combinent ainsi un musée avec un lieu d'enseignement?

R. W. - Parmi les autres musées, Seven Stories à Newcastle est sans doute le meilleur exemple d'exploitation d'archives littéraires à des fins didactiques. D'autres musées le font, mais pas de façon aussi développée. 
C. C.-W. - Vos actions pour promouvoir la lecture et l'écriture prennent des formes multiples. J'ai beaucoup aimé le format du «défi d'écriture de l'été» ("Summer Story Challenge») en six étapes que vous avez proposé cette année. Est-ce qu'il a lieu chaque année?

$R$. W. - Ce programme a été lancé en partie pour pallier la fermeture du musée suite à des inondations. Nous souhaitions maintenir une certaine visibilité sur les réseaux sociaux mais aussi permettre aux familles de tester des idées ensemble pendant les grandes vacances. Les activités proposées ressemblent aux jeux de piste et aux ateliers que nous aurions animés cet été, si bien que le contenu du défi porte sur la vie et les œuvres de Roald, et utilise les alentours du village de Great Missenden et l'archive pour que ce défi soit vraiment spécifique au musée.

Je ne sais pas si nous le referons l'an prochain, tout dépend du succès qu'il rencontrera!

C. C.-W. - Cela fait plusieurs années maintenant que vous travaillez sur ces archives : qu'est-ce qui vous a le plus marquée?
$R$. W. - Roald Dahl était un perfectionniste, qui retravaillait ses textes encore et encore jusqu'à ce que chaque mot soit juste. Il avait la ferme conviction que le goût de la lecture est le meilleur atout que l'on puisse donner à un enfant car cela les instruit, les amuse et les nourrit sur le plan émotionnel tout au long de leur vie.

On retrouve dans ses livres pour la jeunesse cette volonté de divertir les enfants, de les amuser et de les amener à poursuivre leur lecture. Il était le meilleur critique de son propre travail, et réécrivait ses textes jusqu'à ce que chaque mot et chaque phrase le satisfassent et qu'il ait la certitude que son jeune lecteur ne s'ennuierait pas.

D'un point de vue personnel, voir à quel point il était passionné par l'écriture a été à la fois une leçon d'humilité et une source d'inspiration. S'il y a une chose que je peux dire aux enfants qui visitent l'archive et qui voient ses brouillons tout raturés et réécrits, c'est de ne jamais abandonner après la première tentative : continuez jusqu'à ce que votre texte soit le meilleur possible !

Traduit de l'anglais par Christine Collière-Whiteside 